Rapid Reviews COVID-19

\title{
Review 3: "Quantitative Assessment of Olfactory Dysfunction Accurately Detects Asymptomatic COVID-19 Carriers"
}

Dolores Malaspina ${ }^{1}$

${ }^{1}$ Professor, Icahn School of Medicine at Mount Sinai, Psychiatry, Neuroscience, Genetics Genomics

Published on: Oct 19, 2020

DOI: $10.1162 / 2 \mathrm{e} 3983 f 5 . c 121195 \mathrm{a}$

License: Creative Commons Attribution 4.0 International License (CC-BY 4.0). 


\section{$\underline{\text { RR:C19 Evidence Scale rating by reviewer: }}$}

- Potentially informative. The main claims made are not strongly justified by the methods and data, but may yield some insight. The results and conclusions of the study may resemble those from the hypothetical ideal study, but there is substantial room for doubt. Decision-makers should consider this evidence only with a thorough understanding of its weaknesses, alongside other evidence and theory. Decisionmakers should not consider this actionable, unless the weaknesses are clearly understood and there is other theory and evidence to further support it.

*****************************************

\section{Review:}

After self-reported olfactory symptoms were recognized in persons with COVID-19 the introduction of standardized testing showed the vast majority of cases had olfactory deficits. The olfactory changes can precede other symptoms and may be "the canary in the coal mine" to identify asymptomatic carriers. As such persons can unknowingly spread the virus, olfactory tests are being explored to detect viral carriers as a useful public health tool. The development of useful olfactory testing platforms is thus a high priority in the field.

To address this need, these investigators developed a custom-built olfactometer to precisely measures detectability indices at different odor concentrations and an olfactory matching accuracy score with an array of monomolecular odors. Their method identified impaired detection of very low concentrations of odorants in asymptomatic persons who had tested positive for the SARS-CoV-2 virus and were sequestered.

Their method identified $81 \%$ of pre-symptomatic cases as having reduced odor detection. Their quantification combining odor detection and olfactory matching indicated deficits in $81 \%$ of the asymptomatic cases. This is comparable to the proportion of established cases defined as having olfactory deficits using standard psychophysiological tests. For example, in Monell's analysis of 47 studies, $80 \%$ of COVID-19 patients had olfactory loss using off the shelf scratch and sniff tests. Hopkins et al's (2020) European study found olfactory loss in $86 \%$ of mild to moderate cases and a similar larger study by Lechien (2020) found anosmia in 87\% of such cases. 
Strengths of this study include its focused sample of pre-symptomatic cases with confirmed antibody tests, indicating that the olfactory loss precedes other signs of the infection and the methods capacity to show the deficits at very low concentrations compared to their controls.

Limitations in the study are the complexity and unlikely implementation of their olfactometry set- up and their idiosyncratic scoring methods, which were developed in this small sample of pre-symptomatic cases and control subjects. The positive predictive value of this test will be quite distinct in screening, when the prevalence of infection is far lower, which is not discussed.

The impact of the work would be appreciably enhance had standard off the shelf measures of olfactory also been employed. 